\title{
Fehlanreize durch RxGroups im Risikostrukturausgleich? Eine Erwiderung
}

Zur besseren versicherungstechnischen Erfassung der Risiken im Risikostrukturausgleich (RSA) ist beabsichtigt, zusätzlich zu Alter, Geschlecht und dem Erwerbsminderungsrentenstatus auch Arzneimittelverordnungen und stationäre Diagnosen zur Klassifikation von Versicherten heranzuziehen. In der Januar-Ausgabe dieser Zeitschrift wurde von Malin et al. die Gefahr gesehen, dass von dem Indikator Arzneimittelverordnungen Fehlanreize in der Arzneimittelversorgung ausgehen könnten. Auch der Wissenschaftliche Beirat des BKK-Bundesverbandes (im Weiteren: BKK-Beirat) sieht diese Gefahr. Diese Fehlanreize werden aus einer isolierten Betrachtung der Anreizsituation einer einzelnen Krankenkasse abgeleitet. In einem realistischen Szenario müssten aber auch die Reaktionen anderer Akteure im Gesundheitswesen mit einbezogen werden. In diesem Fall erweist sich die Befürchtung, Krankenkassen könnten zu manipulativen Strategien veranlasst werden, als unbegründet.

\section{Prof. Dr. Gerd Glaeske, Dr. Dirk Göpffarth und Frank Otto}

\section{Einleitung}

Seit Einführung des RSA wird darüber diskutiert, dass die Morbiditätslast durch soziodemographische Kriterien nicht ausreichend präzise erfasst werde und dass eine zielgenauere Zuweisung von Beitragsbedarf notwendig sei. Mit dem RSA-Reformgesetz von 2001 hat der Gesetzgeber dieser Diskussion Rechnung getragen und festgelegt, dass der RSA zukünftig „auf der Grundlage von Diagnosen, Diagnosegruppen, Indikationen, Indikationengruppen, medizinischen Leistungen oder Kombinationen dieser Merkmale" die Morbidität unmittelbar berücksichtigen solle.

Das hierzu vom BMG in Auftrag gegebene Gutachten (Reschke et al. 2005) schlägt vor, neben den bisherigen soziodemographischen RSA-Merkmalen zusätzlich sämtliche ambulant zu Lasten der GKV verordneten Arzneimittelwirkstoffe und sämtliche dokumentierten Krankenhausdiagnosen heranzuziehen. Anhand der Kriterien „Senkung der Anreize zur Risikoselektion“, „Förderung der Qualität und Wirtschaftlichkeit der Leistungserbringung" und "Praktikabilität und Kontrollierbarkeit" wurden vorhandene Klassifikationsmodelle verglichen, wobei das amerikanische Modell „IPHCC + RxGroups“ mit

Prof. Dr. Gerd Glaeske, Zentrum für Sozialpolitik der Universität Bremen, Dr. Dirk Göpffarth, Frank Otto, Bundesversicherungsamt, Bonn.
Abstand am besten abgeschnitten hat. Die Gutachter schlagen vor, dieses Modell in einem prospektiven Verfahren einzuführen. Damit werden die Arzneimittelverordnungen und stationären Diagnosen nicht mit den Ausgaben desselben Jahres verknüpft, sondern mit den Ausgaben des Folgejahres. Ein unmittelbarer Bezug zur tatsächlichen Inanspruchnahme ist daher im prospektiven Verfahren nicht gegeben.

Das vorgeschlagene Arzneimittelklassifikationssystem RxGroups wird von einigen Autoren kritisch gesehen, da dieses „Ansatzpunkte für eine ,manipulative' Steuerung der ärztlichen Verordnungsweise durch Krankenkassen“ (BKK-Beirat 2006: 37) biete, die „manipulativ zur Steigerung der RSA-Zuweisungen genutzt werden“ könnten (Malin et al. 2006: 44). Diese Fehlanreize werden in beiden Beiträgen anhand einiger ausgewählter Beispiele illustriert. Angesichts der Ausgabenentwicklung im Arzneimittelbereich wären solche Fehlanreize in der Tat problematisch, so dass sich zwei Fragestellungen ergeben:

- Wie realistisch ist das entworfene Szenario der Einflussnahme der Krankenkassen auf die ärztlichen Therapieentscheidungen?

- Selbst wenn man sich auf dieses Szenario einließe: Sind solche Fehlanreize dem Klassifikationsmodell immanent oder können sie durch relativ einfache Anpassungen beseitigt werden? 


\section{Anreizdiskussion unter realistischen Rahmen- bedingungen}

\subsection{Wann entstehen Fehlanreize?}

Grundsätzlich können Fehlanreize aus Sicht der Krankenkasse nur dann entstehen, wenn der für die Folgekosten entrichtete Morbiditätszuschlag höher ist als die Vergütung der auslösenden Behandlung, d.h. der Deckungsbeitrag zunimmt. Im prospektiven Verfahren liefe die Krankenkasse Gefahr, die Rechnung an den Leistungserbringer bezahlen zu müssen, aber keinen zusätzlichen Beitragsbedarf zu erhalten, wenn der Versicherte im Folgejahr gar nicht mehr bei der Krankenkasse versichert ist (vgl. Daubenbüchel und Göpffarth 2005).

Im prospektiven Modell verliert auch die häufig heraufbeschworene Gefahr, die Versorgungsneutralität könnte verletzt werden, ihre Gültigkeit. Da die Kosten der auslösenden Krankenhausbehandlung nicht in die Zuschläge fließen, werden ambulante und stationäre Behandlungsformen nur dann - zu Recht - unterschiedlich bewertet, wenn sie unterschiedliche Folgekosten aufweisen. Krankenkassen haben keinen Anreiz darauf einzuwirken, dass jeder Arztbesuch zu einer Arzneimittelverordnung führt, weil nur bei Krankheiten mit signifikanten Folgekosten ein Zuschlag ausgelöst würde. Signifikante Folgekosten sind jedoch nur bei chronisch bzw. langfristig Kranken zu erwarten, und hier ist auch davon auszugehen, dass sie eine medikamentöse Therapie grundsätzlich brauchen.

\subsection{Einfluss der Krankenkassen auf ärztliche Therapie- entscheidungen?}

Sowohl Malin et al. (2006) als auch der BKK-Beirat (2006) führen Beispiele an, in denen der veranlasste Morbiditätszuschlag höher ausfällt als die Ausgaben für das den Zuschlag auslösende Medikament. Sie schließen daraus, dass eine Krankenkasse den Anreiz habe, in diesen Fällen eine Mehrverordnung dieses Medikamentes zu betreiben bzw. zuzulassen. Dabei erkennen sie auch richtig, dass die Krankenkasse von einer generellen Mehrverordnung nicht profitieren würde - dies würde zu einem Rückgang der Zuschläge führen. Vielmehr muss die Krankenkasse eine Mehrverordnung für ihre eigenen Versicherten erreichen, für andere Versicherte aber verhindern: Die Strategie ist nur solange lukrativ, wie „ein kritischer Prozentsatz an in gleicher Weise unwirtschaftlich agierenden Kassen nicht überschritten" wird (BKK-Beirat 2006: 31).

Die Annahme, eine Krankenkasse könne die Verordnungen nur für die eigenen Versicherten manipulieren, vernachlässigt die Reaktionen anderer Akteure im Gesundheitswesen. Eine realistische Diskussion der Anreizeffekte muss aber alle „Spieler“ betrachten:

Ärzte: Ärzte sind zunächst verpflichtet, ihre Patienten nach dem Stand der medizinischen Erkenntnisse zu be- handeln. Dies ist kein Appell an die ärztliche Ethik, sondern ein Hinweis auf berufsständische Pflichten und gegebenenfalls von strafrechtlichem Belang. Zudem wäre der Übergang auf eine intensivere Behandlungsstufe oder ein nicht-indiziertes Medikament in der Regel mit höherem Aufwand und höheren Risiken verbunden. Schließlich hat der Arzt - auch bei Einführung einer morbiditätsorientierten Vergütung - keinen ökonomischen Anreiz, statt der indizierten Arzneimittel teurere Präparate zu verordnen. Er hat aber einen Anreiz, Regresse durch die Verordnung preisgünstiger Arzneimittel zu vermeiden.

Betroffene Krankenkassen: Würde eine Manipulationsstrategie bekannt, wäre ein massiver Vertrauensverlust und eine Abwanderung der Versicherten die Folge. Sollte eine Kasse trotzdem eine solche Strategie versuchen, stünde der hohe Aufwand in keinem Verhältnis zum unkalkulierbaren Ertrag. Die Kosten des unwirtschaftlichen Arzneimittels müssten sofort getragen werden, der Ertrag in Form des höheren Zuschlags käme erst zwei Jahre nach der Behandlung. Der Zuschlag stünde unter dem Vorbehalt, dass der Versicherte bei der Krankenkasse verbleibt, die Höhe des Zuschlags tatsächlich so hoch ausfällt wie zwei Jahre vorher antizipiert, und der „Regulator" nicht in der Zwischenzeit den Klassifikationsalgorithmus ändert.

Andere Krankenkassen: Selbst im unrealistischen Fall, dass es einer Krankenkasse gelänge, sich über eine Absprache mit Ärzten einen finanziellen Vorteil gegenüber anderen Krankenkassen zu verschaffen, wäre es wegen des Wettbewerbs der Kassen untereinander nicht vorstellbar, dass die Krankenkasse diese Strategie unbemerkt und unkommentiert von der Öffentlichkeit über längere Zeit durchhalten könnte. Dabei ist auch zu berücksichtigen, dass kein Arzt ausschließlich die Versicherten nur einer Krankenkasse behandelt. Die Überprüfung der Unwirtschaftlichkeit von Arzneimittelverordnungen eines Vertragsarztes erfolgt in Prüfungsausschüssen gemeinsam durch die Vertreter aller Krankenkassen und der jeweiligen Kassenärztlichen Vereinigung. Systematisch nicht indizierte Verordnungen eines Arztes auf Grund von Absprachen mit einer Krankenkasse würden auch hier auffällig, da die Krankenkassen mit ihren konkurrierenden Interessen hierauf ihre erhöhte Aufmerksamkeit richten werden.

Pharma-Unternehmen: Neben den konkurrierenden Krankenkassen hätten auch benachteiligte pharmazeutische Konkurrenzunternehmen ein Interesse an der Aufdeckung manipulativer Strategien - mit gravierenden Folgen für das Arzt-Patienten- und das KrankenkassenVersicherten-Verhältnis.

Patienten: Der Patient kommt als Akteur in den Betrachtungen von Malin et al. und des BKK-Beirats nicht vor. Dabei ist davon auszugehen, dass Patienten einen Wechsel von einer leichteren hin zu einer nicht-indizierten stärkeren Medikation nicht ohne weiteres akzeptieren werden. So werden z.B. Diabetiker, die bislang mit einer Diät oder einem oralen Antidiabetikum behandelt 
worden sind, nicht ohne weiteres bereit sein, sich Insulin zu spritzen, wenn sich ihre Blutzuckerwerte nicht maßgeblich verschlechtert haben.

Regulator: Auch der „Regulator“, d.h. der Verordnungsgeber oder die Durchführungsbehörde, sind relevante Akteure. Anhand der entstehenden Datengrundlage können kassenindividuell die Verordnungen analysiert werden, um kurzfristig - d.h. noch vor Berechnung der Zuschläge $\mathrm{zu}$ reagieren. Glaeske (2005) ${ }^{1}$ schlägt eine Task-Force vor, die kurzfristig den aktuell anfallenden Anpassungsbedarf bearbeitet und dabei auch kontrolliert, ob auffällige unerwünschte Verordnungsänderungen mit diesem Modell in Verbindung gebracht werden können.

Diese Betrachtung gilt grundsätzlich auch, wenn man eine Weiterentwicklung der GKV hin zu direkten Verträgen mit den Leistungserbringern unterstellt. Zwar ließe sich theoretisch vorstellen, dass eine Krankenkasse mit Ärzten in einem direkten Vertrag eine "Sonderbehandlung" (Malin et al. 2006: 46) für ihre Versicherten vereinbart. Eine solche Behandlung mit unnötig teuren Arzneimitteln kann aber weder geheim bleiben noch ohne eine öffentliche Reaktion, insbesondere bei einer Weiterentwicklung wettbewerblicher Strukturen.

\section{Anpassungsfähigkeit des Klassifikationsmodells}

Die Anpassungsfähigkeit von RxGroups soll hier anhand von zwei Beispielen, die auch von Malin et al. bzw. dem BKK-Beirat angeführt wurden, aufgezeigt werden. Eine systematische und differenzierte Untersuchung der Anpassungsnotwendigkeiten und -möglichkeiten von RxGroups nimmt Glaeske (2005) vor.

Die unangepasste Version von RxGroups klassifiziert verschiedene Arten von Antihypertonika in separaten Gruppen: ACE-Hemmer, Betablocker, Diuretika, Calciumantagonisten und AT-II-Antagonisten. Außerdem gibt es eine Gruppe für Kombinationspräparate. Malin et al. (2006) bemängeln nun, dass bei der in der Einstellungsphase oft vorkommenden Suche nach dem am besten wirksamen und verträglichen Arzneimittel mehrere Morbiditätszuschläge ausgelöst würden. Krankenkassen hätten dann ein Interesse daran, dass verschiedene Wirkstoffe aus möglichst vielen verschiedenen Gruppen verordnet würden und dass weniger preiswerte Kombinationspräparate verordnet würden. Diese Betrachtungsweise lässt allerdings die vorgesehene Anpassung von RxGroups völlig außer Betracht. Glaeske (2005) diskutiert verschiedene Optionen und macht konkrete Vorschläge, wie diese Anreize eliminiert werden können. Für Antihypertonika wird die Zusammenfassung der betroffenen RxGroups als Möglichkeit genannt. Dann bestünde weder der Anreiz, eine bestimmte Substanzklasse zu bevorzugen, noch der Anreiz, auf die Verordnung von verschiedenen Wirkstoffen hinzuwirken, und die Verordnung mehrerer Monopräparate brächte einer Krankenkasse keinen finanziellen Vorteil gegenüber der Verordnung eines Kombinationspräparates. Über die Fusion von Gruppen hinaus stehen weitere Instrumente zur Ver- fügung, beispielsweise die Einführung von Mindestverordnungsmengen und Hierarchisierungen.

Praxisrelevante Fehlanreize durch Morbiditätszuschläge unterstellt auch der BKK-Beirat (2006). Zur Illustration wählen sie das Beispiel Diabetes und behaupten, dass hohe Morbiditätszuschläge zu einer Erhöhung der allgemeinen Behandlungsprävalenz führen. Diese undifferenzierte Darstellung unterscheidet nicht zwischen einer erwünschten Verbesserung der Versorgung von bisher unbehandelten Diabetikern und der den Tatbestand der Körperverletzung erfüllenden, nicht erwünschten Behandlung von Gesunden. Der erstgenannte Effekt, die verbesserte Versorgung chronisch Kranker, ist gerade das Ziel der direkten Morbiditätsorientierung. Er sorgt nicht nur für eine Beseitigung bestehender Versorgungsdefizite, sondern vermeidet auch erhöhte Folgekosten aufgrund eines verschlechterten Gesundheitszustandes. Die Vorstellung, dass ein Arzt einem gesunden Versicherten Insulin verordnet, ist hingegen, wie die Autoren selbst einräumen müssen, absurd. Deshalb wechseln sie an dieser Stelle das Beispiel zu Diclofenac, einem häufig eingesetzten Wirkstoff bei der Behandlung von rheumatoider Arthritis. Da es jedoch auch fiebersenkend wirkt, könnte es auch bei einer mit Fieber einhergehenden relativ harmlosen und preiswerten akuten Erkrankung eingesetzt werden. So könnten Morbiditätszuschläge generiert werden, die die zu erwartenden Ausgaben bei weitem übertreffen. Abgesehen davon, dass Diclofenac nicht Mittel der Wahl zur Fiebersenkung ist und deshalb bei Ärzten eine Hemmschwelle bei der Verordnung mit dieser Indikation bestehen dürfte, lässt sich auch hier wieder zeigen, wie bei der Anpassung des Modells reagiert werden würde. Durch Mindestverordnungsmengen könnte sichergestellt werden, dass der entsprechende $\mathrm{Zu}$ schlag nur noch bei mehrfacher Verschreibung ausgelöst würde. Die einmalige Verordnung würde dann keinen Beitragsbedarf generieren, so dass die Krankenkassen kein Interesse an der Verordnung von Diclofenac bei Fieber hätten.

\section{Niederländischer RSA als Alternative?}

Dass Arzneimittelverordnungen zur Messung der Morbidität im Rahmen eines RSA grundsätzlich geeignet sind, bestreiten weder Malin et al. noch der BKK-Beirat. Dies kann zumindest daraus geschlossen werden, dass am Ende beider Beiträge der niederländische RSA als Alternative zu RxGroups hervorgehoben wird. In den Niederlanden werden neben Alter, Geschlecht, der Region und dem Beschäftigungs- bzw. Sozialversicherungsstatus auch Arzneimittelverordnungen und stationäre Diagnosen herangezogen. Bei den stationären Diagnosen wird mit dem DCG-Modell das Vorläufermodell zum von Reschke et al. (2005) vorgeschlagenen IPHCC-Modell verwendet. Das Pharmamodell - PCG - besitzt neben dem reinen Klassifikationsalgorithmus eine Reihe von Modifikationen, um Anreizproblemen zu begegnen. 
Diese Mechanismen bestehen zum Beispiel in einer Zusammenfassung von Morbiditätsgruppen, Änderungen des Zuordnungsmechanismus oder Mindestverordnungsmengen (Van de Ven et al. 2004: 48ff.). Genau diese Maßnahmen sind bei der Anpassung des RxGroups-Modells an deutsche Verhältnisse vorgesehen (Glaeske 2005: 53ff.). Der angestellte Vergleich zwischen dem PCG- und dem RxGroups-Modell ist daher ein Vergleich zwischen Äpfeln und Birnen. Während das PCG-Modell eine konkrete Anpassung des amerikanischen CDS-Modells für die Anwendung in einem RSA darstellt, handelt es sich bei RxGroups um ein „rohes“ Modell, bei dem diese Anpassungsleistung erst noch erbracht werden muss. Dabei ist die konkrete Anpassung als wichtiger anzusehen als das Startmodell. Am Ende wird ein GKV-Grouper entstehen, der sich vom Ausgangsmodell erheblich unterscheidet. Eine ähnliche Erfahrung wurde mit den DRGs gemacht: In den heutigen G-DRGs sind die ursprünglichen australischen AR-DRGs kaum wiederzuerkennen.

Für das niederländische Modell kann daher nicht die bessere Anreizverträglichkeit als Argument angeführt werden, sondern allenfalls, dass die bei RxGroups noch zu leistende Anpassungsarbeit hier bereits geleistet wurde. Dieses Argument kann aber aus zwei Gründen nicht für die Forderung, das PCG-Modell auch in Deutschland einzuführen, herangezogen werden: Zum einen entspricht die Anpassung an das niederländische Gesundheitssystem nicht einer Anpassung an das deutsche System, so dass auch hier weitere Anpassungsarbeiten notwendig würden. Zum anderen liegt dem PCG-Modell eine besondere Philosophie zugrunde, die einen Einsatz im deutschen RSA kritisch erscheinen lässt.

Der Kontext, in dem niederländische Krankenkassen ihre Leistungen anbieten und in dem daher Morbiditätsrisiken ausgeglichen werden müssen, ist ein anderer als in Deutschland. In den Niederlanden besteht neben der Krankenversicherungspflicht eine einheitliche PflichtGrundversicherung (AWBZ), die die Pflegeversicherung durchführt, aber auch die Kosten schwerwiegender stationärer Aufenthalte, die psychische Gesundheitsfürsorge etc. übernimmt. So liegt die psychiatrische Behandlung, deren Erfassung über Arzneimittel ohne Zweifel Schwierigkeiten bereitet, in den Niederlanden gar nicht in der Verantwortung der Krankenkassen. Darüber hinaus besteht ein Risikopool mit einem Schwellenwert von 12.500 Euro $^{2}$ sowie weitere retrospektive Ausgabenausgleiche, so dass die finanziellen Risiken, die eine Krankenkasse bei schwerwiegenden Erkrankungen tragen muss, sehr viel geringer sind als in Deutschland. Entsprechend geringer sind auch die Anforderungen an die Erklärungskraft eines RSA.

Während das Klassifikationssystem RxGroups den Ansatz verfolgt, das komplette Morbiditäts- und Verordnungsspektrum zu erfassen, konzentrieren sich die $\mathrm{Zu}$ schläge bei PCG auf gesundheitspolitisch ausgewählte Krankheitsbilder und Wirkstoffgruppen. Diese Beschränkung erhöht die Versorgungsfehlanreize für die nicht berücksichtigten Krankheitsbilder - z.B. Hämophilie - und dürfte einen Druck von Betroffenengruppen auf die Politik auslösen, „ihre“ Krankheit zu berücksichtigen. Diese Selektivität ruft bei den Krankenkassen Anreize zur Risikoselektion gegenüber Versicherten hervor, deren Krankheit nicht von PCG erfasst wird. Das eingeschränkte Spektrum an zuschlagsauslösenden Verordnungen wiederum definiert eine Art Positivliste. Bekommt ein Parkinsonpatient den Wirkstoff Ropinirol verordnet, erhält seine Krankenkasse einen Zuschlag. Bekommt er jedoch Cabergolin, geht die Kasse leer aus. Im niederländischen Kontext erklärt sich dies dadurch, dass Cabergolin aufgrund einer expliziten Positivliste nicht erstattungsfähig ist.

Gerade das niederländische Beispiel zeigt, dass die theoretischen Anreizwirkungen, die Malin et al. (2006) RxGroups unterstellen, in der Praxis offensichtlich nicht zum Tragen kommen. Die dort angesprochenen Wirkstoffe Ciclosporin, Topiramat und Lamivudin werden im PCG-Modell ebenso wie in RxGroups zur Identifikation von Organtransplantationen, Epilepsie bzw. HIV herangezogen. Darüber hinaus werden im PCG-Modell beispielsweise Diabetiker nur über die Verordnung von Insulin und nicht über orale Antidiabetika identifiziert. Der theoretische Anreiz für die Krankenkassen, jedem Diabetiker Insulin verordnen zu lassen, führt in den Niederlanden nicht zu verändertem Verordnungsverhalten der Ärzte. ${ }^{3}$ Wenn aber Anreizwirkungen in den Griff zu kriegen sind und die Krankenkassen in Deutschland ein breiteres Krankheitsspektrum versichern, gibt es keinen nachvollziehbaren Grund, das Morbiditätsspektrum nicht zu erweitern, um so die Prognosegüte zu verbessern und damit die Zielgenauigkeit des RSA zu erhöhen.

\section{Schlussfolgerungen}

Bei einer realistischen Betrachtung der Anreizsituation hätten manipulative Strategien von Krankenkassen keine Erfolgsaussichten. Dies liegt nicht nur daran, dass sich kein gleichgerichtetes Interesse von Krankenkassen und Leistungserbringern ergibt, sondern auch daran, dass in einem wettbewerblichen Gesundheitswesen die konkurrierenden Interessen der verschiedenen Akteure schnell zu einer Offenlegung solcher Strategien führen würden. Nicht zuletzt darf die Anpassungsfähigkeit des Klassifikationsmodells und die Möglichkeit, auch kurzfristig auf Fehlentwicklungen zu reagieren, nicht unterschätzt werden. Diese Schlussfolgerung darf auch aus der Erfahrung mit dem niederländischen RSA gezogen werden. Aus der niederländischen Erfahrung darf jedoch nicht geschlossen werden, dass eine Einschränkung des im RSA berücksichtigten Morbiditätsspektrums oder des Verordnungsspektrums geeignete Wege sind.

\section{Literatur}

BKK-Beirat (2006): Finanzielle Effekte von RxGroups-klassifizierten Arzneiverordnungen in einem morbiditätsorientierten Risikostrukturausgleich, Gutachten des Wissenschaftlichen Beirats der betrieblichen Krankenversicherung, Essen. 
Daubenbüchel, R.; D. Göpffarth (2005): Chancen und Risiken einer direkten Morbiditätsorientierung, Die Krankenversicherung 57 (2): 42-46.

Glaeske, G. (2005): Anpassung des Klassifikationsmodells RxGroups an die spezifischen Voraussetzungen in der GKV, Köln, http://www.bmg.bund.de/cln_041/nn_603380/SharedDocs/Publikationen/Forschungsberichte/f-001,templateId=raw, property=publicationFile.pdf/f-001.pdf [Zugriff am 12.04.2006]

Malin, E.-M.; P. Hernold, C. König (2006): Fehlanreize durch arzneimittelbasierte Morbiditätszuschläge im Risikostrukturausgleich, Gesundheits- und Sozialpolitik, 61 (1-2): 43-48.

Reschke, P.; S. Sehlen; G. Schiffhorst; W.F. Schräder; K.W. Lauterbach; J. Wasem (2005): Klassifikationsmodelle für Versicherte im Risikostrukturausgleich, Bundesministerium für Gesundheit und Soziale Sicherung, Forschungsbericht 334, Bonn.

Van de Ven, W.P.M.M ; R.C.J.A van Vliet; L.M. Lamers (2004): Health-Adjusted Premium Subsidies In The Netherlands, Health Affairs 23 (3): $45-55$

\section{Fußnoten}

1 Es ist im übrigen erstaunlich, dass Malin et al. auf diese schon einige Zeit vorliegende Expertise mit keinem Wort eingehen, obwohl sie sich ausführlich mit den Anreizwirkungen von RxGroups beschäftigt.

2 Dieser Risikopool macht auch die von Malin et al. (2006) angestellten Vergleiche der Prognosegüte problematisch. Bei Reschke et al. (2005) ist zu beachten, dass dort die Prognosegüte von PCG anhand eines selbst entwickelten Regressionsansatzes ohne Hierarchien berechnet wurde, in den Niederlanden jedoch ein Zellenansatz zum Einsatz kommt.

3 Auskunft von Prof. van de Ven beim Internationalen Workshop zur Einführung der direkten Morbiditätsorientierung in den Risikostrukturausgleich am 14. April 2005 (Bericht unter http://www.bmg.bund.de/cln 041/nn_603380/SharedDocs/Publikationen/nur_download/f-334-workshop,templateId=raw,property=publicationFile.pdf/f-334-workshop.pdf abrufbar).

\section{Patientenverfügung}

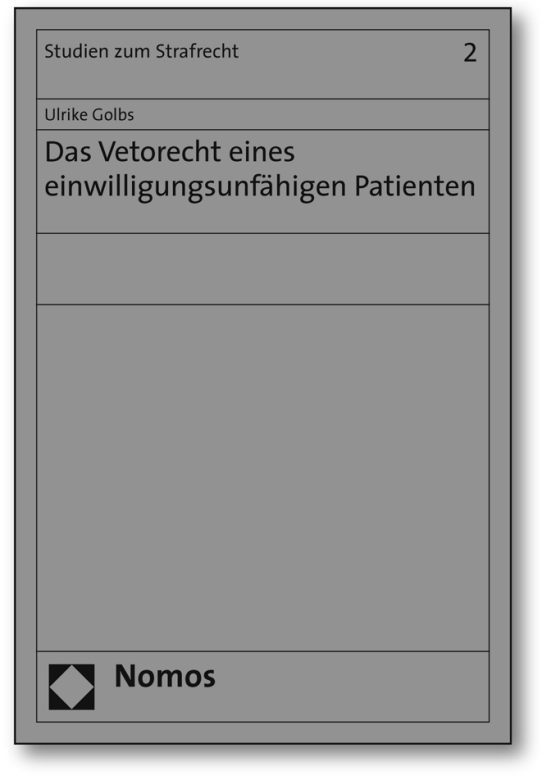

Bitte bestellen Sie bei Ihrer Buchhandlung oder bei: Nomos Verlagsgesellschaft 76520 Baden-Baden | www.nomos.de

\section{Das Vetorecht eines einwilligungsunfähigen Patienten}

Von RAin Ulrike Golbs

2006, 243 S., brosch., 49,- €, ISBN 3-8329-1920-1

(Studien zum Strafrecht, $B d .2$ )

Das Werk untersucht die Frage des Umgangs mit einer Behandlungsverweigerung eines einwilligungsunfähigen Patienten zu einer medizinischen Maßnahme gegen den Willen oder Rat des gesetzlichen Vertreters bzw. Arztes. Dem geht eine eingehende Auseinandersetzung zur Einwilligungsfähigkeit und zur Ernsthaftigkeit eines eingelegten Patientenvetos voran. Für die Einräumung des Vetorechts wird ein Vergleich zu bereits existierenden Regelungen in Spezialgesetzen vorgenommen und die Anwendung auf die medizinische Heilbehandlung hergeleitet. Die Voraussetzungen und Grenzen werden in praxisnaher Weise dargestellt. 